\title{
Simplified exercise test for the initial differential diagnosis of Pneumocystis carinii pneumonia in HIV antibody positive patients
}

Jaume Sauleda, Joaquim Gea, Xavier Aran, M Carmen Aguar, Mauricio Orozco-Levi, Joan M Broquetas

\begin{abstract}
Background - This study was designed to evaluate the usefulness of a simplified exercise test in the differential diagnosis of Pneumocystis carinii pneumonia (PCP).

Methods - Forty five subjects with antibodies against the human immunodeficiency virus (HIV) and pneumonia were included and divided into two groups: those with PCP and those with "other pneumonias" (non-PCP). The test involved pedalling for two minutes on a stretcher bed and was considered positive if $\mathrm{SaO}_{2}$ decreased by at least $3 \%$. Results - During the exercise the mean(SE) $\mathrm{SaO}_{2}$ fell in patients with PCP from $88(4) \%$ to $84(3) \%, p<0.01$, whilst it improved slightly in subjects with nonPCP from 91(1)\% to 93(3)\%, p $<0.05$. Sensitivity was $77 \%$ and specificity $91 \%$. Conclusions - This simple test seems potentially useful for the initial investigation of HIV antibody positive patients with pneumonia.
\end{abstract}

(Thorax 1994;49:112-114)

Pneumocystis carinii pneumonia (PCP) is the most frequent lung infection encountered in patients with AIDS. ${ }^{1}$ The clinical picture is somewhat non-specific, however, and making a definitive diagnosis can be time consuming. A sensitive and specific diagnostic technique which can easily be performed in the emergency department would therefore be useful in the initial management of such patients.

Abnormalities of gas exchange are frequent in patients with PCP and appear to be more evident during exercise. ${ }^{2-4}$ However, ergometric devices are expensive and may be unavailable at admission. This study evaluates the usefulness of a simplified form of the exercise test for the initial diagnosis of PCP in patients with antibodies against HIV.

Dr J G Gea.

Servei de Pneumologia

Hospital del Mar, Pg

Maritim 27, 08003

Barcelona, Spain

Presented in part at the International Conference of the American Thoracic Society, Anaheim, California, 1991

Received 29 March 1993 Returned to authors 10 June 1993

Revised version received 18 August 1993

Accepted for publication

2 November 1993

\section{Methods}

POPULATION

Sixty consecutive subjects with pneumonia, ${ }^{5}$ suspected of being HIV antibody positive, who were admitted to the emergency department at our institution were included in the study. Subjects in whom HIV antibodies were not later identified, non-collaborating patients, those with a history of previous respiratory or cardiovascular diseases, and those with any characteristics that could potentially alter $\mathrm{SaO}_{2}{ }^{6}$ were excluded. Forty five patients were finally included, all of whom began empirical treatment. When diagnosis was established, the subjects were divided into two different subgroups: those with PCP and those with "other pneumonias" (non-PCP). The study was approved by the Committee on Human Research at our institution and all subjects gave their informed consent to the study.

EXERCISE TEST

The exercise test involved making pedalling motions in the air ( 40 cycles/minute) for two minutes on the stretcher bed currently used in the emergency department. Throughout the test $\mathrm{SaO}_{2}$ was continuously recorded by an oximeter (Ohmeda 3740, Louisville, USA). The test was arbitrarily considered as positive if $\mathrm{SaO}_{2}$ decreased by at least 3\% when exercising.

\section{DIAGNOSTIC PROCEDURES}

To classify the patients at least two blood cultures and a fibreoptic bronchoscopic examination (Olympus BF 10, Japan) with bronchoalveolar lavage and telescopic plugged catheter (Mill-Rose Laboratories, Cleveland, Ohio, USA) were performed in all cases. The samples were processed to identify different microorganisms.

STUDY DESIGN

After the patients suspected of being HIV antibody positive and of having pneumonia were admitted, a routine procedure was implemented that included a medical questionnaire, physical examination, and chest radiograph. Patients in whom pneumonia was confirmed then performed the exercise test. Fibreoptic bronchoscopy was carried out within the following 36 hours.
STATISTICAL ANALYSIS

Data are expressed as mean(SE). Paired $t$ tests were used to compare data obtained before and during the exercise. Current definitions for sensitivity, specificity, efficiency, and error of prediction were used. 


\section{Results}

Of the 45 patients included in the study, 36 were intravenous drug abusers.

PCP GROUP

This group comprised 22 patients of mean(SE) age 31 (1) years in whom the chest radiograph showed either a diffuse or localised infiltrate. Patients were moderately hypoxaemic (63(3) $\mathrm{mm} \mathrm{Hg}$ ) breathing room air, and all were treated with either pentamidine or cotrimoxazole immediately after admission. Only four patients, all of whom suffered from severe respiratory failure $\left(\mathrm{PaO}_{2} 40,42,46\right.$, and $50 \mathrm{~mm} \mathrm{Hg}$, respectively) died. During the exercise test $\mathrm{SaO}_{2}$ fell from 88(4)\% to $84(3) \%$ $(p<0.01)$ in this group (figure). The test was positive in 17 subjects including those who died from PCP.

\section{NON-PCP GROUP}

This group consisted of 23 patients of mean(SE) age $28(1)$ years. An organism was identified in 20 cases: Streptococcus pneumoniae (nine), Staphylococcus aureus (four), Haemophilus influenza (three), Legionella pneumophila (two), Pseudomonas aeruginosa (one), and Salmonella typhimurium (one). The radiographic abnormalities were either diffuse or localised, and the degree of hypoxaemia was similar to that of the PCP group (68(3) $\mathrm{mm} \mathrm{Hg}$ breathing room air). Co-trimoxazole, erythromycin, and cephalosporins were the antibiotics mainly used in these patients. Only two subjects (with pneumonia due to $P$ aeruginosa and $L$ pneumophila, respectively) died. The mean(SE) $\mathrm{SaO}_{2}$ improved slightly during exercise from $91(1) \%$ to $93(3) \%, p<0.05)$ in this group (figure). The test was negative - that is, $\mathrm{SaO}_{2}$ did not decrease by at least $3 \%$ - in all but two patients.

The sensitivity of the test for PCP was 77\% while the specificity rose to $91 \%$. The efficiency was thus $84 \%$.

\section{Discussion}

Initial management and empirical treatment of HIV antibody positive patients with pneumonia is sometimes difficult. Making a definitive diagnosis may take hours or days, and alternative diagnostic methods would be desirable. ${ }^{7}$ Lung function tests, isotopic techniques, and

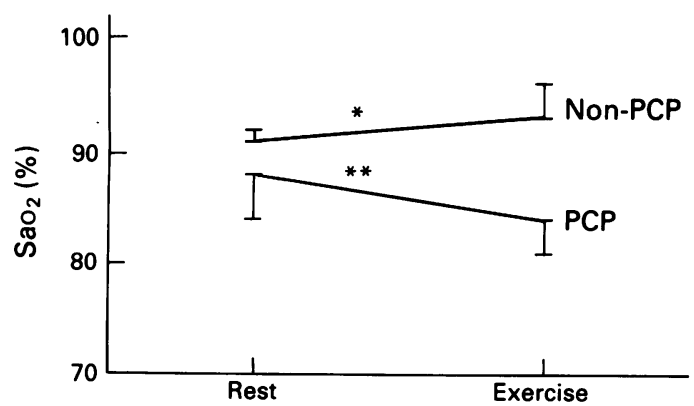

Mean ( $\mathrm{SE}$ ) $\mathrm{SaO}_{2}$ values during rest and exercise in patients with and without Pneumocystis carinii pneumonia (PCP). ${ }^{*} p<0.05 ;{ }^{* *} p<0.01$. measurement of blood lactate dehydrogenase concentrations have been evaluated to date. ${ }^{8-10}$ Nevertheless, not all are available all of the time in all centres; some are relatively expensive, and others are non-specific.

The decrease in $\mathrm{SaO}_{2}$ observed during exercise seems to be relatively specific in patients with $\mathrm{PCP}_{2}{ }^{-4}$ but the ergometric devices required are expensive and may be unavailable both in hospital or at admission. The simplified form of the exercise test used in this study appears to be useful in the differential diagnosis of PCP. Sensitivity was acceptable with an excellent specificity. The test is easily performed in the emergency department using only oximetry. However, it should only be considered as a useful tool and delays in treatment should not be permitted if PCP is suspected on clinical grounds.

Although this test is not completely standardised in terms of level of oxygen uptake or charge, both the intensity (cycles per minute) and duration were equivalent for all the patients.

The decrease in $\mathrm{SaO}_{2}$ observed in patients with PCP, and the slight increase observed in the other group, probably reflect different responses to an increase in cardiac output (QT) resulting from the exercise. In patients with PCP this could have resulted in both a decrease in capillary transit time for red cells and an increase in blood flow to both shunt and low $\dot{\mathrm{V}} \mathrm{A} / \dot{Q}$ units, thereby causing $\mathrm{SaO}_{2}$ to fall. In patients without PCP the increased QT could have augmented the mixed venous oxygen content, thus favouring a better level of $\mathrm{SaO}_{2}$.

The test would probably lose specificity if a differential diagnosis between PCP and other diffuse interstitial pneumonias was attempted, and some sensitivity in localised forms of PCP. However, it is improbable that patients with PCP were included in the non-PCP group, as all but two survived with no adequate treatment against Pneumocystis.

It is also of interest to note the predominance of intravenous drug abusers in the present series, which reflects the current situation in southern European countries and some areas of the USA. Similar studies should be carried out in other groups at risk for HIV infection.

In summary, this simplified form of exercise test seems to be potentially useful for the initial assessment of HIV antibody positive patients with pneumonia. Moreover, it can be carried out easily both inside and outside the hospital.

The authors acknowledge Joseph A Salazar for his help with editing. The study was supported by grants IMIM 1642380 and Metge Matas (C M Palma).

1 Centers for Disease Control. Update: acquired immunodeficiency syndrome (AIDS). MMWR 1986;35:17-21.

2 Smith DE, McLuckie A, Wyatt J, Gazzard B. Severe exercise hypoxemia with normal or near normal X-rays: a feature of Pneumocystis carinii infection. Lancet 1988;ii:1049-51.

3 Faetkenheuer G, Salzberger B, Allolio B, Pothoff G, Schrappre-Baecher $M$. Exercise oximetry for early dianosis of Pneumocystis carinii pneumonia Lancet 1989;28:222.

4 Stover DE, Green RA, Gagliardi AJ. The use of a simple

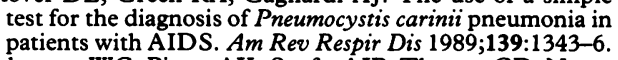

5 Johanson WG, Pierce AK, Sanford JP, Thomas GD. Nosocomial respiratory infection with gram-negative bacilli.

6 Schnapp LM, Cohen NH. Pulse oximetry. Uses and abuses. Chest 1990;98:1244-50. 
7 Millar AB, Mitchell DM. Non-invasive investigation of pulmonary disease in patients positive for the human immunodeficiency virus. Thorax 1990;45:57-61.

8 Shaw RJ, Roussak C, Forster SM, Harris JW, Pinching AJ, Mitchell DM. Lung function abnormalities in patients with the human immunodeficiency virus with and without overt pneumonitis. Thorax 1988;43:436-40.
9 Kramer EL, Sanger JJ, Garay SM. Gallium-67 scans of the chest in patients with acquired immunodeficiency syndrome. F Nucl Med 1987;28:1107-14.

10 Kagawa FT, Kirsch CM, Yenokida GG, Levine ML. Serum lactate dehydrogenase activity in patients with AIDS and Pneumocystis carinii pneumonia. Chest 1988;94:1031-3.

\section{Pulmonary physiology in St Swithin's Hole}

The hospital photographer was an expert speleologist; his photographs of the inside of caves were quite remarkable. One morning he came to the physiology laboratory and asked if we could make him a breathing apparatus to explore under water the further end of a cave in the Mendips. This was before the days of an easily available aqualung. Using a small oxygen cylinder, rebreathing bag and a carbon dioxide absorber we soon had him walking up and down the bottom of the deep end of the local swimming pool.

I had never been caving before and the opportunity to join the party was too good to miss. There were three of us - two experts and me. We arrived at the site in the early morning and walked beside a small stream to where it seemed to disappear into the ground through a grating rather like a mud drain.

"This is where we go in," he said. The entrance was very narrow, and equipped with helmets, lights, food, and the breathing equipment, we squeezed in. The narrow entrance was very wet and we were soon soaked through. The cave's name was obvious.

It was a well used cave with rope ladders down the steep descents, and opened out into spectacular caves with magnificent rock formations. After about two hours we came to the "sump". This was where the cave went under water. It was about 12 feet long and a rope had been put through to assist our passage.

"A deep breath and a pull along the rope and you will be on the other side," he said. What I had not been told was that the floor rose as the roof came down so the opening was quite narrow. I was stuck with my knees on the bottom and my back on the top. The only thing was to go back. I did not panic.

"A second attempt will be easy," I was told, and it was.

We were now in the much less frequented part of the cave and pressed on to the object of our exploration where the cave again went under water. Our intrepid photographer donned his breathing apparatus and, with a rope around his middle, disappeared under water.

"One pull, let out more rope; two pulls, pull me back," he said. We gradually let out more rope. We felt a tug - or was it two? We pulled him back.

"What did you do that for?" he asked. "Let's try again."

He managed to penetrate about 20 feet into the cave with no evidence of where to surface and returned exhausted, mainly because the breathing apparatus could not cope with his hyperventilation under those conditions. A welcome meal and the trek back. I don't think I have ever been quite so tired. Climbing up a rope ladder with a waterfall pouring down your neck after six hours hard work is not easy. Coming out I lost my trousers in the narrow part of the cave. I could not care - my embarrassment of a walk through the village was saved by a thick fog which we found when we surfaced at last.

PETER J D HEAF 